\title{
A new CP-approach for a parallel machine scheduling problem with time constraints on machine qualifications.
}

\author{
Arnaud Malapert \\ Université Côte d'Azur, CNRS, I3S, France \\ arnaud.malapert@unice.fr \\ Margaux Nattaf \\ Univ. Grenoble Alpes, CNRS, Grenoble INP, G-SCOP, 38000 \\ Grenoble, France \\ margaux.nattaf@grenoble-inp.fr
}

October 17, 2019

\begin{abstract}
This paper considers the scheduling of job families on parallel machines with time constraints on machine qualifications. In this problem, each job belongs to a family and a family can only be executed on a subset of qualified machines. In addition, machines can lose their qualifications during the schedule. Indeed, if no job of a family is scheduled on a machine during a given amount of time, the machine lose its qualification for this family. The goal is to minimize the sum of job completion times, i.e. the flow time, while maximizing the number of qualifications at the end of the schedule. The paper presents a new Constraint Programming model taking more advantages of the $\mathrm{CP}$ feature to model machine disqualifications. This model is compared with two existing models: an Integer Linear Programming (ILP) model and a Constraint Programming (CP) model. The experiments show that the new $\mathrm{CP}$ model outperforms the other model when the priority is given to the number of disqualifications objective. Furthermore, it is competitive with the other model when the flow time objective is prioritized.
\end{abstract}

keywords : Parallel Machine Scheduling, Time Constraint, Machine Qualifications, Integer Linear Programming, Constraint Programming. 


\section{Introduction}

Process industries, and specially semiconductor industries, need to be more and more competitive and they are looking for strategies to improve their productivity, decrease their costs and enhance quality. In this context, companies must pay constant attention to manufacturing processes, establish better and more intelligent controls at various steps of the fabrication process and develop new scheduling techniques. One way of doing it is to integrate scheduling and process control [15]. This paper considers such a problem: the integration of constraints coming from process control into a scheduling problem.

Semiconductor fabrication plants (or fabs) have characteristics that make scheduling a very complex issue [7. Typical ones includes a very large number of jobs/machines, multiple job/machine types, hundreds of processing steps, reentrant flows, frequent breakdowns... Scheduling all jobs in a fab is so complex that jobs are scheduled in each workshop separately. In this paper, the focus is on the photolithography workshop, which is generally a bottleneck area. In this area, scheduling can be seen as a scheduling problem on non-identical parallel machines with job family setups (also called s-batching in [7]).

Fabrication processes of semiconductors are very precise and require a high level of accuracy. Reliable equipment are required and valid recipe parameters should be provided. Advanced Process Control (APC) systems ensure that each process is done following predefined specifications and that each equipment is reliable to process different product types. APC is usually associated with the combination of Statistical Process Control, Fault Detection and Classification, Run to Run (R2R) control, and more recently Virtual Metrology [8. The main interest of this paper is to consider, in scheduling decisions, constraints induced by R2R controllers. As shown in the survey paper of [13], R2R control is becoming critical in high-mix semiconductor manufacturing processes.

R2R controller uses data from past process runs to adjust settings for the next run as presented for example in [9] and [3]. Note that a R2R controller is associated to one machine and one job family. In order to keep the R2R parameters updated and valid, a R2R control loop should regularly get data. Hence, as presented in [11, 12], an additional constraint is defined on the scheduling problem to impose that the execution of two jobs of the same family lies within a given time interval on the same (qualified) machine. The value of the time threshold depends on several criteria such as the process type (critical or not), the equipment type, the stability of the control loop, etc. If this time constraint is not satisfied, a qualification run is required for the machine to be able to process again the job family on the machine. This procedure ensures that the machine works within a specified tolerance and is usually time-consuming. In this paper, we assume that qualification procedures are not scheduled either because the scheduling horizon is not suficiently long or because qualification procedures have to be manually performed and/or validated by process engineers. Therefore, maintaining machine qualifications as long as possible is crucial. More precisely, it is important to have as many remaining machine qualifications as possible at the end of the schedule, so that future jobs can also 
be scheduled.

To our knowledge, there are few articles dealing with scheduling decisions while integrating R2R constraints. [2] and [6] study related problems, except that they allow qualification procedures to be performed, the number or the type of machines is different and the threshold is expressed in number of jobs instead of in time. The scheduling problem addressed in this paper has been introduced in [12, where two Integer Linear Programs (ILP) and two constructive heuristics are proposed. More recently, [10, 11] develop a new ILP, modelling problem constraints in a better way. The paper also present one Constraint Programming (CP) model, as well as two improvement procedures of existing heuristics.

In this paper, a new $\mathrm{CP}$ model, which take more advantages of the $\mathrm{CP}$ features, is presented. The main idea of this model is to exploit the fact that once a machine is disqualified, it is until the end of the schedule. The consequence of this is that it is possible to model machine disqualifications more accurately. Then, the performance of this model is compared with the two exact solution methods described in [10. The paper is organized as follows. Section 2 gives a formal description of the problem. Section 3 presents the two models of [10]. Section 4 describes the new CP model and finally, Section 5 provides a detailed comparison of the performance of each model.

\section{Problem description}

Formally, the problem takes as input a set of jobs, $\mathcal{N}=\{1, \ldots, N\}$, a set of families $\mathcal{F}=\{1, \ldots, F\}$ and a set of machines, $\mathcal{M}=\{1, \ldots, M\}$. Each job $j$ belongs to a family and the family associated with $j$ is denoted by $f(j)$. For each family $f$, only a subset of machine, $\mathcal{M}_{f} \subseteq \mathcal{M}$, is able to process a job of $f$. A machine $m$ is said to be qualified to process a family $f$ if $m \in \mathcal{M}_{f}$. Each family $f$ is associated with the following parameters:

- $n_{f}$ denotes the number of jobs in the family. Note that $\sum_{f \in \mathcal{F}} n_{f}=N$.

- $p_{f}$ corresponds to the processing time of jobs in $f$.

- $s_{f}$ is the setup time required to switch the production from a job belonging to a family $f^{\prime} \neq f$ to the execution of a job of $f$. Note that this setup time is independent of $f^{\prime}$. In addition, no setup time is required between the execution of two jobs of the same family.

- $\gamma_{f}$ is the threshold value for the time interval between the execution of two jobs of $f$ on the same machine. Note that this time interval is computed on a start-to-start basis, i.e. the threshold is counted from the start of a job of family $f$ to the start of the next job of $f$ on machine $m$. Then, if there is a time interval $\left.] t, t+\gamma_{f}\right]$ without any job of $f$ on a machine, the machine lose its qualification for $f$.

The objective is to minimize both the sum of job completion times, i.e. the flow time, and the number of qualification looses or disqualifications. Note that the interest of minimizing the number of disqualifications comes from the fact 


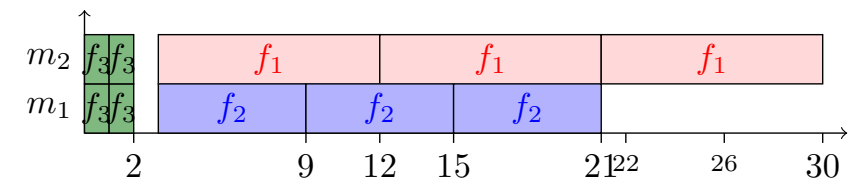

(a) An optimal solution for the flow time objective

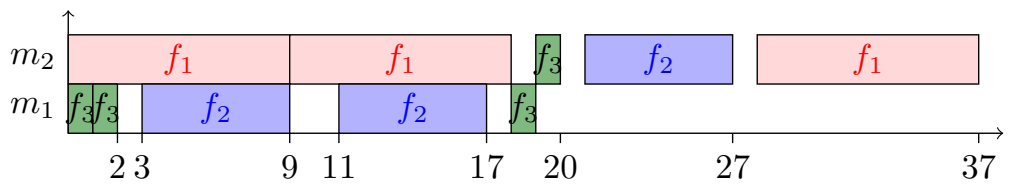

(b) An optimal solution for qualification losses

Figure 1: Two solution examples for PTC.

that, even if the time horizon considered is relatively small, the problem is solved in a rolling horizon. Hence, it is interesting to preserve machine qualifications for future jobs. In addition, it is relevant to consider that a machine cannot lose its qualification for a family after the end of the schedule. Thus, this assumption is made in the remaining of the paper. This problem, introduced in [12], is called the scheduling Problem with Time Constraints (PTC). An example of PTC together with two feasible solutions is now presented.

\section{Example 1}

Consider the following instance with

$N=10, M=2$ and $F=3$ :

\begin{tabular}{|c|ccccl|}
\hline$f$ & $n_{f}$ & $p_{f}$ & $s_{f}$ & $\gamma_{f}$ & $\mathcal{M}_{f}$ \\
\hline 1 & 3 & 9 & 1 & 25 & $\{2\}$ \\
2 & 3 & 6 & 1 & 26 & $\{1,2$ \\
3 & 4 & 1 & 1 & 21 & $\{1,2$ \\
\hline
\end{tabular}

Figure 11 shows two feasible solutions. The first solution, described by Figure 1a, is optimal in terms of flow time. For this solution, the flow time is equal to $1+2+9+15+21+1+2+12+21+30=114$ and the number of qualification losses is 3 . Indeed, machine $1\left(m_{1}\right)$ loses its qualification for $f_{3}$ at time 22 since there is no job of $f_{3}$ starting in interval ]1,22] which is of size $\gamma_{3}=21$. The same goes for $m_{2}$ and $f_{3}$ at time 22 and for $m_{2}$ and $f_{2}$ at time 26.

The second solution, described by Figure 16, is optimal in terms of number of disqualifications. Indeed, in this solution, none of the machines loses their qualifications. However, the flow time is equal to $1+2+9+17+19+9+18+20+$ $27+37=159$. This shows that the flow time and the number of qualification losses are two conflicting criteria. Indeed, to maintain machine qualifications, one need to regularly change the job family executed on machines. This results in Nate also that disqualifications may qfcur after the last job on the machine. For example, in Figure $1 \mathrm{1a}, \mathrm{m}_{1}$ become disqualified for $f_{3}$ at time 22 whereas the last job scheduled on $m_{1}$ finishes at time 21 . However, no disqualifications can occur after the makespan $C_{\max }$.

Remark 1 (Bound on the makespan) In the remaining of the paper, the following upper and lower bound on the makespan are defined. The upper bound used is the same as in [12], i.e. $T=\overline{C_{\max }}=\sum_{f \in \mathcal{F}} n_{f} \cdot\left(p_{f}+s_{f}\right)$. A trivial lower bound is $\underline{C_{\max }}=\left\lceil\left(\sum_{f \in \mathcal{F}} n_{f} * p_{f}\right) / M\right\rceil$. 
Remark 2 (Bi-objective optimization) In [12], PTC is studied using a weighted sum of the flow time and number of disqualifications. The weight associated to the flow time is $\alpha$ and is always equal to 1 . The weight associated with the number of disqualifications is $\beta$ and is set to 1 when the priority is given to the flow time and to $N \cdot T$ when the priority is given to the number of disqualifications. In this paper, two objective modelling are considered: the weighted sum and the lexicographical order. The weighted sum is used in the ILP model in all cases and in the CP model only when the minimization of the flow time is prioritized. The lexicographical order is used in the CP model when the priority is given to the number of disqualifications.

\section{Existing Models}

This section describes the two exact methods developped in [10. First, the ILP is described and then, the $\mathrm{CP}$ model. Note that both $\mathrm{CP}$ model described in this paper use the CP Optimizer (CPO) framework. Indeed, CPO allow us to handle in an efficient way precedence constraints and optional jobs. Furthermore, constraint propagation of these type of constraints is very efficient with CPO.

\subsection{ILP model}

The ILP model in [10] is an improvement of two existing models introduced in [12]. The first ILP model of [12] relies on a job-based formulation. Indeed, in this model, a variable $x_{j, t}^{m}$ is defined for each job $j$, each machine $m$ and each time $t$. This variable is then equal to 1 if and only if job $j$ starts at time $t$ on machine $m$. However, in a solution, there is no need to know which job start at which time on which machine. Indeed, only the family of the job is important. Hence, a family-based model is developed in 12 (IP2) and improved in [10] (IP3).

In the family-based model, a variable $x_{f, t}^{m}$ is introduced for each family $f \in$ $\mathcal{F}$, each machine $m \in \mathcal{M}_{f}$ and each time $t \in \mathcal{T}=\{0, \ldots, T-1\}$, with $T$ the upper bound on the makespan (see Remark 1). This variable is set to one if and only if one job of $f$ starts at time $t$ on machine $m$. Therefore, the number of binary variables is reduced compared to the job-based model.

Similarly, a set of variable $y_{f, t}^{m}$ is used to model disqualifications. This variable is set to 1 only if family $f$ lose its qualification on machine $m$ at time $t$. However, in (IP2), it may occur that a machine become disqualified after $C_{\max }$. Thus, in (IP3), another variable set $Y_{f}^{m}$ is defined to model the fact that a machine becomes disqualified for a family before $C_{\max }$.

$$
\begin{aligned}
& \operatorname{min.} \alpha \cdot \sum_{f \in \mathcal{F}} C_{f}+\beta \cdot \sum_{f \in \mathcal{F}} \sum_{m \in \mathcal{M}} Y_{f}^{m} \\
& \sum_{m \in \mathcal{M}_{f}} \sum_{t=0}^{T-p_{f}} x_{f, t}^{m}=n_{f}
\end{aligned}
$$

$\forall f \in \mathcal{F}$ 


$$
\begin{aligned}
& \sum_{m \in \mathcal{M}_{f}} \sum_{t=0}^{T-p_{f}}\left(t+p_{f}\right) \cdot x_{f, t}^{m} \leq C_{f} \\
& n_{f} \cdot x_{f^{\prime}, t}^{m}+\sum_{\tau=t-p_{f}-s_{f^{\prime}}+1}^{t} x_{f, \tau}^{m} \leq n_{f} \\
& y_{f, t}^{m}+\sum_{\tau=t-p_{f}+1}^{t} x_{f, \tau}^{m} \leq 1 \\
& y_{f, t}^{m}+\sum_{\tau=t-\gamma_{f}+1}^{t} x_{f, \tau}^{m} \geq 1 \quad \forall f \in \mathcal{F}, \forall t \geq \gamma_{f} \in \mathcal{T}, \forall m \in \mathcal{M}_{f} \\
& y_{f, t-1}^{m} \leq y_{f, t}^{m} \quad \forall f \in \mathcal{F}, \forall t \in \mathcal{T}, \forall m \in \mathcal{M}_{f}
\end{aligned}
$$

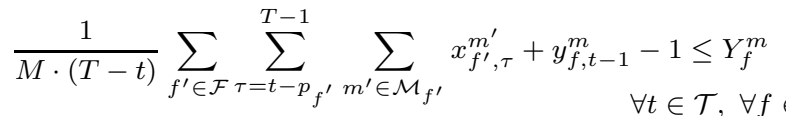

$$
\begin{aligned}
& x_{f, t}^{m} \in\{0,1\} \\
& \forall t \in \mathcal{T}, \forall f \in \mathcal{F}, \forall m \in \mathcal{M}_{f} \\
& y_{f, t}^{m} \in\{0,1\} \\
& Y_{f}^{m} \in\{0,1\} \\
& \forall t \in \mathcal{T}, \forall f \in \mathcal{F}, \forall m \in \mathcal{M}_{f} \\
& \forall t \in \mathcal{T}, \forall f \in \mathcal{F}, \forall m \in \mathcal{M}_{f} \\
& \forall f \in \mathcal{F}, \forall m \in \mathcal{M}_{f}
\end{aligned}
$$

The objective of the model is described by (11). It is expressed as the weighted sum of the flow time and the number of disqualifications. Constraints (2) ensure that all jobs are executed. Constraints (3) is used to compute the completion time of family $f$, i.e. the sum of completion time of all jobs of $f$. Constraints (4) ensure that jobs of $f$ and jobs of $f^{\prime}$ does not overlap and that the setup times are satisfied. Constraints (5) are used to model both the fact that the execution of two jobs of the same family cannot occur simultaneously and the fact that a machine has to be qualified to process a job. Constraints (6) make sure that if no jobs of family $f$ start on $m$ during an interval $\left.] t-\gamma_{f}, t\right]$, then $m$ becomes disqualified for $f$ at time $t$. Constraints (7) maintain the disqualification of the machine once it becomes disqualified. Finally, Constraints (8) ensure that it is no longer necessary to maintain a qualification on a machine if there is no job which starts on any machine in the remainder of the horizon, i.e. $\frac{1}{M \cdot(T-t)} \sum_{f^{\prime} \in \mathcal{F}} \sum_{\tau=t-p_{f^{\prime}}}^{T-1} \sum_{m^{\prime} \in \mathcal{M}_{f^{\prime}}} x_{f^{\prime}, \tau}^{m^{\prime}}=0$.

The number of variables of the model is $F \cdot M \cdot(2 T+1)$ and the number of constraints is at most $2 F+T \cdot M \cdot\left(4 F+F^{2}\right)$.

\subsection{CP model}

In this part, the CP model defined in [10] is described. The first part of the model concerns the modelling of a classical parallel machine scheduling problem (PMSP) with setup time and the second part deals with the modelling of the disqualifications.

\subsubsection{The parallel machine scheduling problem with setup time}

The PMSP with setup time can be modeled using optional (or not) interval variables introduced by [4, 5]. An (optional) interval variable $J$ is associated with four variables: a start time, st $(J)$; a duration, $d(J)$; an end time, et $(J)$ and a binary execution status $x(J)$, equal to 1 if and only if the interval variable 
is present in the final solution. If the job $J$ is executed, it behaves as a classical job that is executed on its time interval, otherwise it is not considered by any constraint.

In the considered scheduling problem, a job $j$ of family $f$ can be scheduled on any machine belonging to $\mathcal{M}_{f}$. Therefore, a set of optional interval variables alt $J_{j, m}$ is associated with each job $j$ and each machine belonging to $\mathcal{M}_{f(j)}$. The domain of such variables is $\operatorname{dom}\left(\right.$ alt $\left._{j, m}\right)=\left\{[s t\right.$, et $) \mid[s t, e t) \subseteq[0, T), s t+p_{f(j)}=$ et\}. Furthermore, a non-optional interval variable, $j o b s_{j}$ is associated with each job $j$. Its domain is $\operatorname{dom}\left(j o b s_{j}\right)=\left\{[s t, e t) \mid[s t, e t) \subseteq[0, T), s t+p_{f(j)}=e t\right\}$.

To model the PMSP with setup time, the following two sets of global constraints is used [14].

Alternative constraints Introduced in 4, this constraint models an exclusive alternative between a bunch of jobs.

$$
\text { alternative }\left(\text { jobs }_{j},\left\{\text { alt }_{j, m} \mid m \in \mathcal{M}_{f(j)}\right\}\right) \quad \forall j \in \mathcal{N}
$$

It means that when $j o b s_{j}$ is executed, then exactly one of the alt $J_{j, m}$ jobs must be executed, i.e. the one corresponding to the machine $m$ on which the job is scheduled. Furthermore, the start date and the end date of $j o b s_{j}$ must be synchronised with the start and end date of the alt $J_{j, m}$ jobs. However, if $j o b s_{j}$ is not executed, none of the other jobs can be executed. In our model, $j o b s_{j}$ is a mandatory job. This constraint models the fact that each job must be executed on one and only one machine.

No-Overlap constraints An important constraint is that jobs cannot be executed simultaneously on the same machine. It is a unary resource constraint. Each machine can then be used by only on job at a time. To model this feature, we use noOverlap constraints. This constraint ensures that the execution of several interval variables do not overlap. It can also handle the setup time. Let $S$ be the matrix of setup times of the problem, i.e. $\left(S_{f^{\prime}, f}\right)= \begin{cases}0 & \text { if } f=f^{\prime} \\ s_{f} & \text { oherwise }\end{cases}$ Then, the following noOverlap constraint makes sure that, for all pairs of jobs $(i, j)$ s.t. $m \in \mathcal{M}_{i} \cap \mathcal{M}_{j}$, either the start of alt $J_{j, m}$ occurs after the end of alt $J_{i, m}$ plus $s_{f(j)}$ or the opposite:

$$
\text { noOverlap }\left(\left\{\text { alt }_{j, m} \mid \forall j \text { s.t. } m \in \mathcal{M}_{f(j)}\right\}, S\right) \quad \forall m \in \mathcal{M}
$$

The exact semantic of this constraint is presented in [5].

Additional ordering constraints The authors of [10 add a non-mandatory set of constraints to the model. Indeed, the model is correct without these constraints but adding them remove many symmetry in the model. The constraint order the start of jobs belonging to the same family.

$$
\text { startBeforeStart }\left(\text { jobs }_{j}, \text { jobs }_{j}^{\prime}\right) \quad \forall j, j^{\prime} \in \mathcal{N}, j>j^{\prime}, f\left(j^{\prime}\right)=f(j)
$$

\subsubsection{Modelling of the number of disqualifications}

In the model of [10], disqualifications are modelled as optional interval variables. The variable will be present in the final solution if and only if, the machine 
became disqualified for the family. The start time of the variable corresponds to the time at which the machine becomes disqualified. Therefore, a set of optional interval variable, $d i s q_{f, m}$, of length 0 is defined for each family $f$ and each machine $m$ such that $m \in \mathcal{M}_{f}$. The domain of these variables is $\operatorname{dom}\left(\operatorname{disq}_{f, m}\right)=$ $\left\{[s t, e t) \mid[s t, e t) \subseteq\left[\gamma_{f}, T\right)\right.$, st $=$ et $\}$. In addition, the model will use a $C_{\max }$ interval variable of length 0 modelling the end time of the last job executed on all machine, i.e. the end of the schedule. Its domain is $\operatorname{dom}\left(C_{\max }\right)=$ $\{[s t, e t) \mid[s t, e t) \subseteq[0, T)$, st $=e t\}$.

Then, the constraints used to model machine disqualifications are stated below. The first set of constraints model the fact that each job has to be executed before $C_{\max }$.

$$
\text { endBeforeStart }\left(\text { jobs }_{j}, C_{\max }\right) \quad \forall j \in \mathcal{N}
$$

Another set of constraints ensures that no job of a family $f$ is scheduled on $m$ if $m$ is disqualified for $f$, i.e. after the $d i s q_{f, m}$ job.

$$
\text { startBeforeStart }\left(\text { alt }_{j, m}, \operatorname{disq}_{f(j), m}, \gamma_{f(j)}\right) \quad \forall j \in \mathcal{N}, \forall m \in \mathcal{M}_{f(j)}
$$

Finally, the following constraints sets enforce a machine to become disqualified if no job of family $f$ is scheduled on $m$ during an interval of size $\gamma_{f}$. Indeed, the first set state that if a job of $f$ is scheduled on $m$, either there is another job of $f$ scheduled on $m$ less than $\gamma_{f}$ units of time later, or the machine become disqualified, or the end of the scheduled $\left(C_{\max }\right)$ is reached.

$$
\begin{array}{r}
x\left(\operatorname{alt}_{j, m}\right) \Rightarrow \bigvee_{\substack{j^{\prime} \neq j \\
f(j)=f\left(j^{\prime}\right)}}\left(\operatorname{st}\left(\operatorname{alt}_{j^{\prime}, m}\right) \leq t_{j, m}\right) \vee\left(\operatorname{st}_{\left.\left(\operatorname{disq}_{f(j), m}\right)=t_{j, m}\right) \vee\left(C_{\max } \leq t_{j, m}\right)}\right. \\
\forall j \in \mathcal{N}, \forall m \in \mathcal{M}_{f(j)}
\end{array}
$$

with $t_{j, m}=s t\left(a l t J_{j, m}\right)+\gamma_{f(j)}$. The second set of constraints ensures that if no job of $f$ is scheduled on $m$, then $m$ becomes disqualified for $f$.

$$
\begin{aligned}
\bigvee_{\substack{j \in \mathcal{N} \\
f(j)=f\left(j^{\prime}\right)}}\left(\operatorname{st}_{\left.\left(\text {alt } J_{j, m}\right) \leq \gamma_{f}\right) \vee\left(\operatorname{st}\left(\operatorname{disq}_{f(j), m}\right)=\gamma_{f}\right) \vee\left(s t\left(C_{\max }\right)\right.} \leq \gamma_{f}\right) & \\
& \forall f \in \mathcal{F}, \forall m \in \mathcal{M}_{f}
\end{aligned}
$$

\subsubsection{Objective function}

In the considered problem, the objective is to minimize both the flow-time and the number of disqualifications. In this CP model, the flow-time can be expressed as flowTime $=\sum_{j \in \mathcal{N}} e t\left(j o b s_{j}\right)$ and the number of disqualifications as $n b$ Disq $=\sum_{f \in \mathcal{F}} \sum_{m \in \mathcal{M}} x\left(\right.$ disq $\left._{f, m}\right)$.

\subsubsection{Model size}

The number of variables of the model is at most $N \cdot(M+1)+M \cdot F+1$ and the number of constraints is at most $N^{2}+2 N+M \cdot(1+2 N+F)$.

\section{New CP Model}

This section presents a new CP model that can be used to solve PTC. As said earlier, PTC can be decomposed into two sub-problems: a PMSP with setup time and a machine qualifications problem. The model described in this section 
uses the same idea as in [10] to formulate the first sub-problem of PTC. However, to model the machine qualification sub-problem a novel approach is developed modelling qualifications as resource.

The first part of this section described the difference of modelling of the PMSP between the model of Section 3.2 and the model of this section. The second part is dedicated to the machine qualification sub-problem.

In the model, the two following assumptions are made. First, it is assumed that jobs of the same family have consecutive index in $\mathcal{N}$. More precisely, with $n_{f}$ the number of jobs in family $f$ then jobs with index in $\left\{1, \ldots, n_{1}\right\}$ belong to family 1 , jobs with index in $\left\{n_{1}+1, \ldots, n_{1}+n_{2}\right\}$ are jobs of family 2 , etc. The second assumption made in the model is that it is equivalent to consider the threshold either on an end-to-end basis or on a start-to-start basis. Indeed, if a job of family $f$ starts at time $t$ on $m$, another job of $f$ has to start before $t+\gamma_{f}$. This is equivalent to: if a job of family $f$ ends at time $t+p_{f}$ on $m$, another job of $f$ has to end before $t+p_{f}+\gamma_{f}$. Therefore, the model consider the threshold on an end-to-end basis. The motivation for this second assumption will be given later in the section.

\subsubsection{The parallel machine scheduling problem with setup time}

As for the model of Section 3.2 , the parallel machine scheduling problem with setup time is model using interval variables $j o b s_{j}, \forall j \in \mathcal{N}$, and optional interval variables alt $J_{j, m}$. The constraints used are the same and, therefore, are not described in this section.

Cumulative constraints The model is also reinforced by considering the set of machines as a cumulative resource of capacity $M$. Indeed, each job consumes one unit of resource (one machine) during its execution and the total capacity of the resource (total number of machines available) is $M$. This is expressed using the global constraint cumulative [1].

$$
\text { cumulative }\left(\left\{\left(\text { jobs }_{j}, 1\right) \mid \forall j \in \mathcal{N}\right\}, M\right)
$$

Makespan modelling As for the previous model, the makespan of the scheduling is needed to model machine disqualifications. The constraints presented in this section concern the link between the makespan and the PMSP. A constraint linking the makespan with the number of disqualifications will be presented later in the paper.

Unlike the previous model, the makespan is modeled here as an interval variable starting at time 0 and spanning the execution of all jobs. This is modelled using span constraints. Introduced in [5], this constraint states that an executed job must span over a set of other executed jobs by synchronising its start date with the earliest start date of other executed jobs and its end date with the latest end date. It is expressed by the following constraints:

$$
\begin{array}{r}
\operatorname{span}\left(C_{\max },\left\{j_{\text {jobs }} \mid \forall j \in \mathcal{N}\right\}\right) \\
\operatorname{st}\left(C_{\max }\right)=0
\end{array}
$$

In addition, the size of the interval has to be between the upper and the lower bound on the makespan defined in Remark 1. 


\subsubsection{Machine qualifications problem}

In this section, the model for the machine qualifications problem is described. The main idea of the model is that, each time a job of family $f$ is scheduled on a machine $m$, a qualification interval of size $\gamma_{f}$ will occur right after. This interval "models" the fact that machine $m$ remains qualified for family $f$ until, at least, the end of the interval. To model this feature, optional interval variable are used. Indeed, for each job $j$ and each machine $m \in \mathcal{M}_{f(j)}$, an optional interval variable, $q u a l_{j, m}$, of size $\gamma_{f(j)}$ and taking its value in $\left\{0, \ldots, T+\max _{f} \gamma_{f}\right\}$. is created. Then, a variable $q_{u a l}, m$ will be present in the solution only if alt $J_{j, m}$ is present and will start at the end of alt $J_{j, m}$. This is expressed by the following set of constraints.

$$
\begin{array}{ll}
x\left(\operatorname{alt}_{j, m}\right)=x\left(\text { qual }_{j, m}\right) & \forall j \in \mathcal{N}, \forall m \in \mathcal{M}_{f(j)} \\
\operatorname{endAtStart}\left(\text { alt }_{j, m}, \text { qual }_{j, m}\right) & \forall j \in \mathcal{N}, \forall m \in \mathcal{M}_{f(j)}
\end{array}
$$

Hence, a job of $f$ can only be scheduled on $m$ during a qualification interval of $f$ on $m$. This is modeled using cumulative functions. A cumulative function $Q_{f, m}$ counts, at each time $t$, the number of qualification intervals for $(f, m)$ in which $t$ is. If the number of qualification intervals for $(f, m)$ is greater than 1 , then a job of $f$ can be scheduled on $m$. Otherwise, the number of interval is zero and $m$ is disqualified for $f . Q_{f, m}$ is expressed as:

$$
Q_{f, m}=\text { pulse }\left(0, \gamma_{f}+p_{f}, 1\right)+\sum_{\substack{j \in \mathcal{N} \\ f(j)=f}} \sum_{m \in \mathcal{M}_{f(j)}} \text { pulse }\left(q u a l_{j, m}, 1\right)
$$

Indeed, at the beginning of the scheduled, the machine is qualified from time 0 to $\gamma_{f}+p_{f}$. In addition, each time an interval variable $q u a l_{j, m}$ is scheduled, $Q_{f(j), m}$ increases by one. Then, when a job of $f$ is scheduled on $m, Q_{f, m}$ has to be greater than one and one can show that $Q_{f, m}$ is always smaller than $n_{f}+1$.

$$
\operatorname{always} \operatorname{In}\left(Q_{f(j), m}, \text { alt }_{j, m}, 1, n_{f(j)}+1\right) \quad \forall j \in \mathcal{N}, \forall m \in \mathcal{M}_{f(j)}
$$

Example 2 (Example of cumulative function) Considering the instance of Example 1. The cumulative function $Q_{f_{3}, m_{1}}$ corresponding to Figure 16 is described by Figure 2.

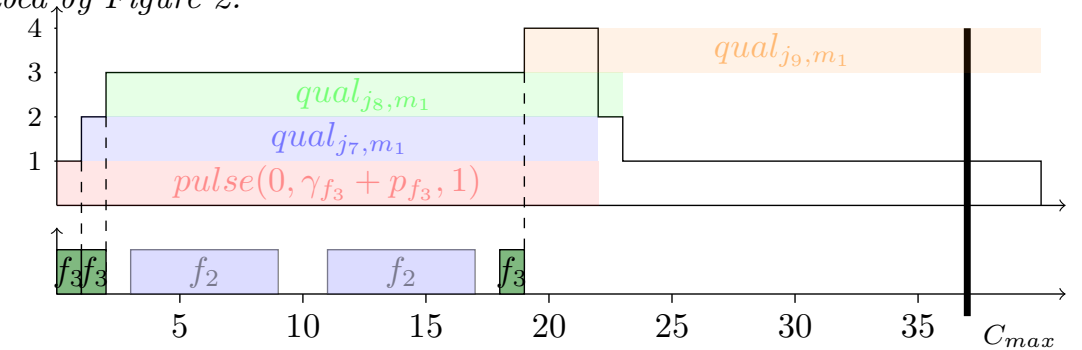

Figure 2: Example of cumulative function to model qualifications.

Each time a job of $f_{3}$ ends, the value of the function $Q_{f_{3}, m_{1}}$ increases by one and decreases when the qualification interval ends. While the value of $Q_{f_{3}, m_{1}}$ is greater than one, it is possible to schedule jobs of $f_{3}$ on $m_{1}$. Here, $Q_{f_{3}, m_{1}}$ is always greater than one for $t \in\left[0, C_{\max }\right)$ meaning that $m_{1}$ remains qualified for $f_{3}$ at the end of the schedule. 
Machine disqualifications Another dummy optional interval variable set, end $Q_{f, m}$, is introduced to check if a machine has been disqualified for a family during the schedule. The variable is present in the final solution only if the machine is still qualified at the end of the schedule. In this case, the variable start at time 0 , ends at time $C_{\max }+p_{f}$ and the function $Q_{f, t}$ has to be greater than one during the whole execution of job $e n d Q_{f, m}$ (otherwise, the machine has been disqualified).

$$
\begin{array}{ll}
\operatorname{st}\left(\operatorname{end} Q_{f, m}\right)=0 & \forall f \in \mathcal{F}, \forall m \in \mathcal{M}_{f} \\
\text { endAtEnd }\left(C_{\text {max }}, \operatorname{end}_{f, m}, p_{f}\right) & \forall f \in \mathcal{F}, \forall m \in \mathcal{M}_{f} \\
\text { alwaysIn }\left(Q_{f, m}, \text { end }_{f, m}, 1, n_{f}+1\right) & \forall f \in \mathcal{F}, \forall m \in \mathcal{M}_{f}
\end{array}
$$

Note that this modelling is possible because the threshold were considered on an end-to-end basis. Otherwise, the use of constraints alwaysIn is not possible.

\subsubsection{Ordering constraints}

The following sets of constraint (partially) order variables in the solution. These (partial) ordering is used to break symmetries in the model. Recalling that it is assumed that jobs of the same family have consecutive index in $\mathcal{N}$. Then constraints (28) state that $j o b s_{j-1}$ has to start before $j o b s_{j}$ and constraints (29) that the maximum time lag between these jobs is $\gamma_{f(j)}$. Constraints (30) order jobs that cannot be executed in parallel. Indeed, job $j$ can overlap at most $M_{f(j)}-1$. Hence, job $j-M_{f(j)}$ cannot overlap job $j$ and has to end before. Constraints (31) ensure that the qualification interval corresponding to job $j$ is separated from the qualification interval of $j-1$ by at least the duration of the job. Finally, constraints (32) model the fact that, on a machine $m$, jobs of a same family are ordered, i.e. smaller index scheduled first.

$$
\begin{aligned}
& \left.{\operatorname{startBeforeStart}\left(\text { jobs }_{j-1}, j_{\text {obs }}\right)}\right) \quad \forall j \in \mathcal{N} \text { s.t. } f(j)=f(j-1) \\
& \text { startBeforeSart }\left(\text { jobs }_{j}, j_{\text {obs }}{ }_{j-1},-\gamma_{f(j)}\right) \quad \forall j \in \mathcal{N} \text { s.t. } f(j)=f(j-1) \\
& \text { endBeforeStart }\left(j o b s_{j-M_{f(j)}}, j o b s_{j}\right) \quad \forall j \in \mathcal{N} \text { s.t. } f(j)=f\left(j-M_{f(j)}\right) \\
& \text { startBeforeStart }\left(\text { qual }_{j-1, m}, \text { qual }_{j, m}, p_{f(j)}\right) \\
& \forall m \in \mathcal{M}_{f(j)}, \forall j \in \mathcal{N} \text { s.t. } f(j)=f(j-1) \\
& \text { endBeforeStart }\left(\text { alt }_{i, m}, \text { alt }_{j, m}\right) \quad \forall m \in \mathcal{M}_{f(j)}, \forall i<j \in \mathcal{N} \text { s.t.f } f(i)=f(j)
\end{aligned}
$$

\subsubsection{Objective function}

The objective function is modeled using two integer variables: flowTime $\epsilon$ $\left\{\underline{C_{\max }}, \ldots, \overline{C_{\max }}\right\}$ and qualified $\in\left\{1, \ldots, \sum_{f \in \mathcal{F}} M_{f}\right\}$. The expressions of these variables are given below:

$$
\begin{aligned}
& \text { flowTime }=\sum_{j \in \mathcal{N}} \text { et }\left(j o b s_{j}\right) \\
& \text { qualified }=\sum_{f \in \mathcal{F}} \sum_{m \in \mathcal{M}_{f(j)}} x\left(\text { end }_{f, m}\right)
\end{aligned}
$$

Then, the objective is expressed as a sum, i.e. (flow - qual), or using the lexicographical order, e.g. lex(-qual, flow). Note that, in this model, the number of machine qualified at the end of the schedule is maximized which is equivalent to minimize the number of machine becoming disqualified during the schedule. 


\subsubsection{Model size}

The number of variables of the model is at most $N \cdot(2 M+1)+M \cdot F+3$ and the number of constraints is at most $N^{2} \cdot M+4 N+M \cdot(1+4 N+3 F)+6$.

\section{$5 \quad$ Experiments}

This section starts with the presentation of the instances used in the experiments (Section [5.1). Then, the general framework of the experiments is described in 5.2. Finally, the three model presented in the paper are used to solve the instances and the results are compared and analysed (Section [5.3).

\subsection{Instance generation}

The benchmark instances used to perform our experiments are extracted from [10]. In this paper, 19 instance sets are generated with different number of jobs $(N)$, machines $(M)$, family $(F)$ and qualification schemes. Each of the instance sets is a group of 30 instances and are generated as follows.

In each generated instances, each family can be executed by at least one machine and each machine is qualified to process at least one job family. Furthermore, since short thresholds may lead to very quick machine disqualifications, the time thresholds of job families are chosen sufficiently large compared to their associated processing times, i.e. $\max _{f \in \mathcal{F}} p_{f} \leq \min _{f \in \mathcal{F}} \gamma_{f}$. Then, to ensure diversity, each set of instances contains 10 instances with small threshold (corresponding to duration needed to process one to two jobs of another family than $f$ ), 10 with medium threshold (two to three jobs) and 10 with large threshold (three to four jobs). In addition, setup times are not chosen too large so that the risk of disqualifying a machine due to a setup time insertion is "acceptable", i.e. $\max _{f \in \mathcal{F}} s_{f} \leq \min _{f \in \mathcal{F}} p_{f}$.

Table 1 presents the parameters of the different instance sets. In the first rows, the different number of jobs $N$ is given, number of machines $M$ is describedby the second row and number of families $F$ is detailed in the third row. Note that each triplet $(n, m, f)$ corresponds to 30 instances. Among those instances, at least $99,5 \%$ are feasible. Indeed, experiments in [10] show that only one 60-job instance and two 70-job instances have an unknown status. For all other instances, at least one of the algorithms presented in [10] is able to find a feasible solution.

\begin{tabular}{|c|c|c|c|c|c|c|c|c|c|c|c|c|c|c|c|c|c|c|}
\hline$N$ & \multicolumn{6}{|c|}{20} & \multicolumn{6}{|c|}{30} & 40 & 50 & 6 & & & 70 \\
\hline$M$ & & & & & & & & & & & 4 & 5 & & & 3 & & & 4 \\
\hline$F$ & 4 & 5 & 2 & 3 & 4 & 5 & 2 & 3 & 4 & 5 & 4 & 5 & 3 & 3 & 4 & 5 & 5 & 4 \\
\hline
\end{tabular}

The instances generatedable 1: Instance chafacteristics to industrial instances. However, due to the complexity of the problem, it is important to first analyse and compare the results of the three models described in this paper. Finding 
good solutions for industrial instances is a real challenge and is an important research direction for future work.

\subsection{Framework}

The experiment framework is defined so the following questions are addressed: Question 1. Which model is the best at finding a feasible solution, proving the optimality and/or finding good upper bounds (especially when solving large instances)?

Question 2. Does the performance of a model change depending on the objective function or on the time limit?

The models are implemented using IBM Ilog Optimization Studio 12.8. That is CPLEX 12.8 for the ILP model and CP Optimizer 12.8 for CP models. All the experiments were led on a computer running on Ubuntu 16.04.5 with $32 \mathrm{~GB}$ of RAM and one Intel Core i7-3930K $3.20 \mathrm{GHz}$ processors (6 cores). Furthermore, two time limits are used in the experiments: 30 and 600 seconds.

Two heuristics are used to find solutions which are used as a basis for the models. These heuristics are called Scheduling Centric Heuristic and Qualification Centric Heuristic [10]. The goal of the first heuristic is to minimize the flow time while the second one tries to minimize the number of disqualifications.

In the following of the section ILP model, $\mathrm{CP}_{O}$ model and $\mathrm{CP}_{N}$ model denotes respectively the ILP model of section 3.1 the previous CP model described in Section 3.2 and the new CP model detailled in Section 4 Furthermore, to describe the performance of the different models, the following indicators are used in the table of Section 5.3. \% sol. described the percentage of instances for which feasible solution is found; \%opt. shows the percentage of instances for which the optimality is proven; \%vbs provides the percentage of instances for which the model is the virtual best solver, i.e. has found the best solution compared to others; \#dis. gives the average number of disqualified machines and finally, obj. is used to show the average of the sum of the flowtime and the number of disqualified machines.

In addition, a bold value in the table means that the corresponding indicator has the best values among its row, i.e. compared to the one of the others model.

\subsection{Comparison of the three models}

This section aims at comparing the results of the three models. First, the results are described for the tight time limit, i.e. 30 seconds. Then, the results with the 600-seconds time limits are given.

\subsubsection{0-seconds time limit}

Minimizing the number of disqualifications over the flow time Table2 gives indicators for the three models solved using the lex (-qual, flow) objective with 30-seconds time limit. 


\begin{tabular}{|c|c|c|c|c|c|c|}
\hline \multirow[b]{2}{*}{$N$} & \multicolumn{4}{|c|}{ ILP model } & $\mathrm{CP}_{O}$ model & $\mathrm{CP}_{N}$ model \\
\hline & $\%$ so & $l . \%$ opt &.$\% v b$ & $s \# d i s$ & \%sol.\%opt.\%vbs\#dis. & \%sol.\%opt.\%vbs\#dis. \\
\hline 20 & 100 & 54.4 & 55.6 & 1.1 & $\mathbf{1 0 0} 69.486 .1 \quad \mathbf{0 . 6}$ & 10082.290 .60 .6 \\
\hline 30 & 97.2 & 21.7 & 23.3 & 3.1 & $\mathbf{9 9 . 4} 51.1 \quad 59.4 \quad 1.4$ & $98.9 \quad 56.771 .11 .2$ \\
\hline 40 & 100 & 23.3 & 26.7 & 0.9 & $\begin{array}{lllll}100 & 63.3 & 63.3 & 0.6\end{array}$ & 10083.390 \\
\hline 50 & 100 & 0 & 6.7 & 2.9 & $\begin{array}{lllll}100 & 33.3 & 36.7 & 1.4\end{array}$ & 10056.773 .30 .8 \\
\hline 60 & 88.3 & 0 & 0 & 7.5 & $\begin{array}{llll}\mathbf{9 0} & 8.3 & 33.3 & 3.4\end{array}$ & 21.756 .72 .8 \\
\hline 70 & 86.7 & 0 & 0 & 9.5 & $\mathbf{9 1 . 1} 4.4 \quad 41.1 \quad 5$ & 91.115 .652 .24 .1 \\
\hline
\end{tabular}

Table 2: Lexicographic minimization of the disqualified machines and the flowtime within 30 seconds.

Table 2 shows that the ILP model finds less feasible solutions than the CP models. Furthermore, the ILP model does not scale well for large instances. Indeed, the ILP model is never the VBS and its average number of disqualified machines is very high for the largest instances compared to the CP models.

On the other hand, the $\mathrm{CP}_{N}$ model obtains better results than the $\mathrm{CP}_{O}$ model. Indeed, the percentage of proof of optimality is higher with $\mathrm{CP}_{N}$ model. The model is also more often the VBS regardless of the instance size. Furthermore, the difference between the average numbers of qualified machines of both model increases with the instance size. This shows that the $\mathrm{CP}_{N}$ model scales better than the $\mathrm{CP}_{O}$ model.

Minimizing the flow time over the number of disqualifications Table 3 gives indicators for the three models solved using the $($ flow - qual) objective with 30 -seconds time limit.

\begin{tabular}{|c|c|c|c|c|c|c|c|c|c|c|c|c|}
\hline \multirow[b]{2}{*}{$N$} & \multicolumn{4}{|c|}{ ILP model } & \multicolumn{4}{|c|}{$\mathrm{CP}_{O}$ model } & \multicolumn{4}{|c|}{$\mathrm{CP}_{N}$ model } \\
\hline & $\%$ sol & $\%$ or & $\% v b s$ & $o b j$. & $\%$ sol & $\% o$ & $\% v b s$ & $\overline{o b j .}$ & $\%$ sol & $\% o p t$ & $\% v b s$ & $\overline{o b j .}$ \\
\hline 20 & 100 & 96.7 & 97.8 & 334. & 100 & 0 & 87.8 & 334.8 & 100 & 65.6 & 90 & 334. \\
\hline 30 & 97.8 & 69.4 & 72.2 & 782. & 99.4 & 0 & 71.1 & 770 & 98.9 & 24.4 & 57.8 & 766 \\
\hline 40 & 100 & 90 & 90 & 1536 & 100 & 0 & 93.3 & 1530 & 100 & 60 & 100 & 1529 \\
\hline 50 & 100 & 60 & 70 & 2265 & 100 & 0 & 76.7 & 2159 & 100 & 10 & 73.3 & 2151 \\
\hline 60 & 88.3 & 5 & 8.3 & 3228 & 90 & 0 & 50 & 2792 & 90 & 0 & 36.7 & 2805 \\
\hline 70 & 86.7 & 4.4 & 5.6 & 4256 & 90 & 0 & 52.2 & 3583 & 91.1 & 0 & 43.3 & 3562 \\
\hline
\end{tabular}

Table 3: Weighted sum minimization of the flowtime and number of disqualified

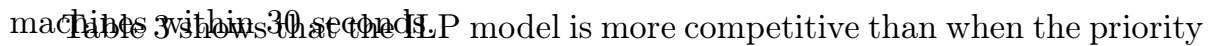
is given to the number of disqualifications. Indeed, despite the fact that it finds a little less feasible solutions than the CP Models, it is better at proving the optimality of its solution. However, the ILP model does not scale well as shown by the high objective values for the largest instances.

On the other hand, the $\mathrm{CP}_{O}$ model is the most efficient for finding good upper bounds, but completely fails at proving the optimality of its solution. The $\mathrm{CP}_{N}$ model proves optimality less often than the ILP model. However, 
it is only slightly dominated by the $\mathrm{CP}_{O}$ model in terms of being the VBS. However, the $\mathrm{CP}_{N}$ model still have the lowest objective values.

\subsection{2 $600-$ seconds time limit}

Table 4 gives indicators for the three models solved using both lexicographic and weighted sum minimization with 30 -seconds and 600-seconds time limit. Only challenging instances with 60 jobs are used to save computation time.

For the lexicographic minimization, the $\mathrm{CP}_{N}$ model confirms its predominance. For all three models, the percentages of solved instances remain constant, the percentages of optimality proof only slightly increase, and the average numbers of disqualified machines significantly decrease.

For the weighted sum minimization, the ILP model becomes the best model. The percentages of solved instances and optimality proof significantly improve and the model often becomes the VBS. Nevertheless, the $\mathrm{CP}_{N}$ model model has the best average objective.

Most of the times, the low improvements of the number of solved instances or optimality proofs suggest that the solvers is subject to thrashing and therefore cannot diversify the search.

\begin{tabular}{|c|c|c|c|c|c|c|c|c|c|}
\hline \multirow[b]{2}{*}{$t$} & \multicolumn{3}{|c|}{ ILP model } & \multicolumn{3}{|c|}{$\mathrm{CP}_{O}$ model } & \multicolumn{3}{|c|}{$\mathrm{CP}_{N}$ model } \\
\hline & $\%$ sol.\%o &.$\% v$ & s\#dis. & $\%$ so & $1 . \%$ opt &.$\% v b s \# d i s$. & $\%$ so &.$\% 0$ & $\%$ vbs \#dis. \\
\hline $30 \mathrm{~s}$ & $88.3 \quad 0$ & 0 & 7.5 & 90 & 8.3 & $33.3 \quad 3.4$ & 90 & & 56.72 .8 \\
\hline $600 \mathrm{~s}$ & 90 & 3.3 & 4.2 & 90 & 11.7 & 28.32 .9 & 90 & & 61.72 .2 \\
\hline$t$ & $\%$ sol.\%ol &.$\% v$ & $j j$ & $\% s$ & $1 . \% c$ & $b s$ obj. & $\%$ & 07 & $\% v b s$ obj. \\
\hline $30 \mathrm{~s}$ & $88.3 \quad 5$ & 8.3 & 3228 & 90 & 0 & $\begin{array}{ll}50 & 2792\end{array}$ & 90 & 0 & 36.72805 \\
\hline $600 \mathrm{~s}$ & 98.355 & 75 & 2873 & 90 & 0 & 33.32755 & 90 & 0 & $33.3 \mathbf{2 7 4 4}$ \\
\hline
\end{tabular}

Table 4: Weighted sum and leximin minimization over instances of 60 jobs Githiccomedussions and further work

A parallel machine scheduling problem was studied where some Advanced Process Control constraints are integrated: minimal time constraints between jobs of the same family to be processed on a qualified machine to avoid losing the qualification. Two criteria to minimize are considered: the sum of completion times and the number of disqualifications.

For this problem, a new CP model was proposed. This model improves the modelling of machine disqualifications. Indeed, when the number of disqualifications is prioritized, this model is better than the existing methods (ILP model and $\mathrm{CP}_{O}$ model) in terms of objective value and in terms of optimality proof. However, when the flow time is prioritized, the performance of the model is less impresive. In this case, the $\mathrm{CP}_{O}$ model tends to have better performance for small-time limit and the ILP model performs better in case of larger time limit.

Experiment results show that a good CP model needs to make some improvements on the modelling and/or the solving of the parallel machine scheduling 
problem with the flow time objective. Interesting research directions include the improvement of variable bounds, specially the makespan. It also includes the study of good relaxations of the problem to enhance the performance of constraint programming models.

Another relevant research perspective consists in scheduling jobs on a longer time horizon, where lost qualifications could be automatically recovered after a given qualification procedure. Qualification procedures, requiring time on machines, would then also be scheduled.

\section{References}

[1] Beldiceanu, N., Carlsson, M., Rampon, J.X.: Global constraint catalog (revision a) (01 2012)

[2] Cai, Y., Kutanoglu, E., Hasenbein, J., Qin, J.: Single-machine scheduling with advanced process control constraints. Journal of Scheduling 15(2), 165-179 (Apr 2012). https://doi.org/10.1007/s10951-010-0215-8, https://doi.org/10.1007/s10951-010-0215-8

[3] Jedidi, N., Sallagoity, P., Roussy, A., Dauzere-Peres, S.: Feedforward runto-run control for reduced parametric transistor variation in cmos logic $0.13 \mu \mathrm{m}$ technology. IEEE Transactions on Semiconductor Manufacturing 24(2), $273-279$ (2011)

[4] Laborie, P., Rogerie, J.: Reasoning with conditional timeintervals. In: Proceedings of the Twenty-First International Florida Artificial Intelligence Research Society Conference, May 15-17, 2008, Coconut Grove, Florida, USA. pp. 555-560 (2008), http://www.aaai.org/Library/FLAIRS/2008/flairs08-126.php

[5] Laborie, P., Rogerie, J., Shaw, P., Vilím, P.: Reasoning with conditional time-intervals. part II: an algebraical model for resources. In: Proceedings of the Twenty-Second International Florida Artificial Intelligence Research Society Conference, May 19-21, 2009, Sanibel Island, Florida, USA (2009), http://aaai.org/ocs/index.php/FLAIRS/2009/paper/view/60

[6] Li, L., Qiao, F.: The impact of the qual-run requirements of APC on the scheduling performance in semiconductor manufacturing. In: Proceedings of 2008 IEEE International Conference on Automation Science and Engineering(CASE). pp. 242-246 (2008)

[7] Moench, L., Fowler, J.W., Dauzère-Pérès, S., Mason, S.J., Rose, O.: A survey of problems, solution techniques, and future challenges in scheduling semiconductor manufacturing operations. Journal of Scheduling pp. 117 (2011), http://dx.doi.org/10.1007/s10951-010-0222-9, 10.1007/s10951010-0222-9 
[8] Moyne, J., del Castillo, E., Hurwitz, A.M.: Run-to-Run Control in Semiconductor Manufacturing. CRC Press, 1 edn. (2000)

[9] Musacchio, J., Rangan, S., Spanos, C., Poolla, K.: On the utility of run to run control in semiconductor manufacturing. In: Proceedings of 1997 IEEE International Symposium on Semiconductor Manufacturing Conference. pp. 9-12 (1997)

[10] Nattaf, M., Dauzère-Pérès, S., Yugma, C., Wu, C.H.: Parallel machine scheduling with time constraints on machine qualifications, Manuscript submitted for publication.

[11] Nattaf, M., Obeid, A., Dauzère-Pérès, S., Yugma, C.: Méthodes de résolution pour l'ordonnancement de familles de tâches sur machines parallèles et avec contraintes de temps. In: 19ème édition du congrès annuel de la Société Française de Recherche Opérationnelle et d'Aide à la Décision, ROADEF 2018

[12] Obeid, A., Dauzère-Pérès, S., Yugma, C.: Scheduling job families on nonidentical parallel machines with time constraints. Annals of Operations Research 213(1), 221-234 (Feb 2014). https://doi.org/10.1007/s10479-012$1107-4$

[13] Tan, F., Pan, T., Li, Z., Chen, S.: Survey on run-to-run control algorithms in high-mix semiconductor manufacturing processes. IEEE Transactions on Industrial Informatics 11(6), 1435-1444 (2015)

[14] Wolf, A.: Constraint-based task scheduling with sequence dependent setup times, time windows and breaks. In: GI Jahrestagung (2009)

[15] Yugma, C., Blue, J., Dauzère-Pérès, S., Obeid, A.: Integration of scheduling and advanced process control in semiconductor manufacturing: review and outlook. Journal of Scheduling 18(2), 195-205 (Apr 2015). https://doi.org/10.1007/s10951-014-0381-1, https://doi.org/10.1007/s10951-014-0381-1 